\title{
Impact of histone deacetylase 1 and metastasis-associated gene 1 expression in esophageal carcinogenesis
}

\author{
TOMOHARU MIYASHITA ${ }^{1}$, HIDEHIRO TAJIMA ${ }^{1}$, MASAYOSHI MUNEMOTO ${ }^{1}$, FURHAWN A. SHAH ${ }^{2}$, \\ JOHN W. HARMON ${ }^{2}$, TOSHIFUMI WATANABE ${ }^{1}$, MASATOSHI SHOJI ${ }^{1}$, KOICHI OKAMOTO ${ }^{1}$, \\ SHINICHI NAKANUMA ${ }^{1}$, SEISHO SAKAI ${ }^{1}$, JUN KINOSHITA ${ }^{1}$, ISAMU MAKINO ${ }^{1}$, \\ KEISHI NAKAMURA ${ }^{1}$, HIRONORI HAYASHI ${ }^{1}$, KATSUNOBU OYAMA ${ }^{1}$, MASAFUMI INOKUCHI ${ }^{1}$, \\ HISATOSHI NAKAGAWARA ${ }^{1}$, HIROYUKI TAKAMURA ${ }^{1}$, ITASU NINOMIYA ${ }^{1}$, HIROHISA KITAGAWA ${ }^{1}$, \\ SACHIO FUSHIDA $^{1}$, KENICHI MUKAISHO ${ }^{3}$, TAKASHI FUJIMURA ${ }^{1}$ and TETSUO OHTA ${ }^{1}$ \\ ${ }^{1}$ Department of Gastroenterological Surgery, Kanazawa University Hospital, Kanazawa, Ishikawa 920-8641, Japan; \\ ${ }^{2}$ Department of Surgery, Johns Hopkins Bayview Medical Center, Johns Hopkins University School of Medicine, Baltimore, \\ MD 21224, USA; ${ }^{3}$ Department of Pathology, Shiga University of Medical Science, Otsu, Shiga 520-2192, Japan
}

Received September 25, 2013; Accepted April 24, 2014

DOI: $10.3892 / 01.2014 .2176$

\begin{abstract}
Animal models are important for the development of novel therapies for esophageal cancer. Histone deacetylase 1 (HDAC1)/metastasis-associated gene (MTA1) complexes inhibit p53 acetylation and thus, inhibit p53-induced apoptosis. The aim of the present study was to evaluate HDAC1 and MTA1 expression in esophageal carcinogenesis in rats. The rats underwent a total gastrectomy followed by esophagojejunostomy to induce chronic duodenal content reflux esophagitis. The rats were sacrificed sequentially at 20,30, 40 and 50 weeks post-surgery and the esophagi were examined. Immunohistochemical analysis was conducted to assess the expression and localization of HDAC1 and MTA1. At 20 weeks post-surgery, squamous proliferative hyperplasia and Barrett's metaplasia (BM) were observed. While, adenocarcinoma-associated BM and squamous cell carcinoma were observed at 30-50 weeks post-surgery. The nuclear expression of HDAC1 and MTA1 was observed in all of the stages of squamous carcinogenesis and adenocarcinogenesis, although not in the normal esophageal epithelium. The expression of
\end{abstract}

Correspondence to: Dr Tomoharu Miyashita, Department of Gastroenterological Surgery, Kanazawa University Hospital, 13-1 Takaramachi, Kanazawa, Ishikawa 920-8641, Japan

E-mail: tomoharumiya@gmail.com

Abbreviations: ESCC, esophageal squamous cell carcinoma; EADC, esophageal adenocarcinoma; HDAC, histone deacetylases; MTA, metastasis-associated gene; GERD, gastroesophageal reflux disease

Key words: metastasis-associated gene, histone deacetylases, esophageal carcinogenesis, esophageal adenocarcinoma, Barrett's esophagus
HDAC1 and MTA1 may be involved in duodenoesophageal reflux-induced neoplastic transformation of the esophageal mucosa into cancer cells with squamous and adeno differentiation.

\section{Introduction}

Esophageal squamous cell carcinoma (ESCC) is the dominant type of esophageal cancer worldwide (1). However, the incidence of esophageal adenocarcinoma (EADC) has been rapidly increasing in the Western world over the last 50 years, particularly in western males $(2,3)$. The etiology of the increase in the incidence of EADC remains obscure and has prompted further investigation into this clinical issue.

The rapid increase of EADC in Western countries has occurred in parallel with an increased prevalence of gastroesophageal reflux disease (GERD) (4,5). Diet and lifestyle alterations in the Western world have been associated with an increased prevalence of obesity and hiatal hernias, which are known risk factors for GERD and esophageal cancer $(5,6)$. A previous study proposed that EADC develops via a sequence of events into GERD (7). Specifically, gastroduodenal content reflux from GERD induces inflammation-mediated hyperplasia and metaplasia, and subsequently dysplasia and EADC.

Studies have also determined that duodeno-esophageal or duodeno-gastro-esophageal reflux induces the sequential development of EADC in surgical rat models (8-10). These cancerous changes occur without the use of any exogenous carcinogens. The rat model demonstrated the histopathological sequence of events from GERD to EADC as an inflammation-metaplasia-dysplasia-adenocarcinoma (ADC) sequence. Furthermore, a recent study has established a correlation between the quantity of reflux and the likelihood of developing EADC and ESCC (11).

The pathogenesis of reflux-induced duodenoesophageal carcinoma and the molecular changes in gene expression, which drives esophageal carcinogenesis in rats, have recently 
been addressed (12). The potential role of histone deacetylase (HDAC) 1 and metastasis-associated gene (MTA) 1 in esophageal carcinogenesis remains unclear and has not been investigated in depth.

$M T A$ is a newly discovered family of cancer progression-associated genes and their encoded products (13). The expression of MTA1 and its encoded protein, MTA1, have been found to correlate with the malignant properties of numerous human cancers, including cancer of the esophagus (14), breast (15), pancreas (16), colon (17), stomach (18), liver (19) and prostate (20).

Histone acetyltransferase (HAT)- and HDAC-induced alterations of the chromatin structure have been implicated in the regulation of gene transcription, as well as in the process of carcinogenesis $(21,22)$.

Chromatin histone and non-histone proteins are the protein targets for HDAC deacetylation via nucleosome remodeling and histone deacetylation (NuRD) complexes containing MTA proteins. The p53 tumor suppressor protein was the first non-histone protein reported to be deacetylated by MTA protein-containing NuRD complexes (23). The HDAC1/MTA1 complexes exert deacetylation activity against p53 protein in human non-small cell carcinoma and human hepatoma cells. In addition, the complexes have been found to inhibit p53-induced apoptosis by attenuating the transactivation function of p53 $(18,24)$.

To improve the understanding of esophageal carcinogenesis in humans, animal models mimicking this tumorigenic process are particularly powerful tools. The use of experimental animal models is an effective method to understand the developmental mechanisms underlying carcinogenesis. The current study utilized a surgically induced rat reflux model of esophageal carcinogenesis. The rat surgical reflux model provided the opportunity to record the expression of proteins encoded by the HDACl and MTAl genes in each stage of carcinogenesis, and to observe the effects on cell proliferation and carcinogenesis. In addition, the model was advantageous as it enabled examination of the expression of the HDACl and MTAl genes in all stages of esophageal carcinogenesis, including squamous hyperplasia, squamous dysplasia, squamous cell carcinoma (SCC), Barrett's esophagus, ADC and adenosquamous carcinoma (ASC). By improving the understanding of the expression of HDAC1 and MTA1 in esophageal carcinogenesis, targeted esophageal cancer chemotherapy may be developed.

The aim of the present study was to assess HDAC1 and MTA1 expression in a surgical rat model of esophageal carcinogenesis.

\section{Materials and methods}

Experimental animals. In total, 50 Wistar male rats, weighing $\sim 250 \mathrm{~g}$, were used in the present study. The animals were housed three per cage and maintained at a constant room temperature of $22 \pm 3^{\circ} \mathrm{C}$, in $55 \pm 5 \%$ humidity under a 12 -h light-dark cycle. The rats were fed standard solid chow (CRF-1; Charles River Laboratories Japan, Inc., Yokohama, Japan) and tap water that was free of carcinogens. The study was approved by the Institutional Animal Care and Use Committee of the Graduate School of Medical Science, Kanazawa University (AP-111868; Kanazawa, Japan).
Surgical procedures. Following a 24-h fast, an upper abdominal incision was made under diethyl ether inhalation anesthesia. The surgical procedures were performed to induce duodenoesophageal reflux following total gastrectomy of each rat as previously reported (10)

Specimen extraction. The animals were sacrificed by diethyl ether inhalation and the abdomen was opened. A ligature was placed around the afferent and efferent jejunal loop proximal to the esophagojejunal anastomosis. The esophagus was ligated at the level of the thyroid cartilage via a thoracotomy. The esophagus and anastomosed jejunum were subsequently removed.

Pathological assessment. The excised organs were washed with $10 \%$ formalin, spread and pinned on a cork plate with the mucosal side facing upwards. Following fixation of the organs with $10 \%$ formalin solution for at least $24 \mathrm{~h}$, the esophagus was cut into slices along the longitudinal axis at 3-mm intervals and embedded in paraffin. Next, $5 \mu \mathrm{m}$-thick sections of each embedded paraffin block were prepared for histological analysis with hematoxylin and eosin staining.

Immunohistochemistry. For the immunohistochemical staining, the Dako Envision system (Dako, Carpinteria, CA, USA), which uses dextran polymers conjugated with horseradish peroxidase, was employed to avoid any endogenous biotin contamination. The sections were deparaffinized in xylene and rehydrated in a graded ethanol series. The endogenous peroxidase was blocked by immersing the sections in $3 \% \mathrm{H}_{2} \mathrm{O}_{2}$ in $100 \%$ methanol for $20 \mathrm{~min}$ at room temperature. Antigen retrieval was achieved by microwaving the sections at $95^{\circ} \mathrm{C}$ for $10 \mathrm{~min}$ in $0.001 \mathrm{M}$ citrate buffer ( $\mathrm{pH} \mathrm{6.7).} \mathrm{After}$ blocking the endogenous peroxidase, the sections were incubated with Protein Block Serum-Free (Dako) at room temperature for $10 \mathrm{~min}$ to block the non-specific staining. The sections were incubated for $2 \mathrm{~h}$ at room temperature with 1:100 diluted mouse antibodies against monoclonal MTA1 (D40D1; Cell Signaling Technology, Inc., Beverly, MA, USA) and polyclonal HDAC1 (ab19845; Abcam, Cambridge, MA, USA). Peroxidase activity was detected with the enzyme substrate, 3-amino-9-ethylcarbazole and for the negative controls, the sections were incubated with Tris-buffered saline (Wako Pure Chemical Industries, Tokyo, Japan) without the primary antibodies. The samples with $\geq 10 \%$ of tumor cells were slightly counterstained with Mayer hematoxylin and considered to show positive staining. Immunohistochemical staining for MTA1/HDAC1 was scored by two of the authors as positive or negative in the epithelium that was under examination. An estimation of the immunohistochemical expression of the markers was determined by counting $\geq 100$ cells in random high-power fields. The frequency of positive cells for each antibody was reported semi-quantitatively as follows: (-), No reaction; $(+)$, mild with $<30 \%$ of positive cells; $(++)$, moderate with $30-60 \%$ of positive cells; and (+++), marked with $>60 \%$ of positive cells. A positive expression was defined as the staining of $>30 \%$ of the cancer cells $(++$ or +++$)$.

Definition of pathological findings. The histological findings in the esophagus were classified into the following four categories: 
Table I. Outcome and histological findings.

Postoperative week

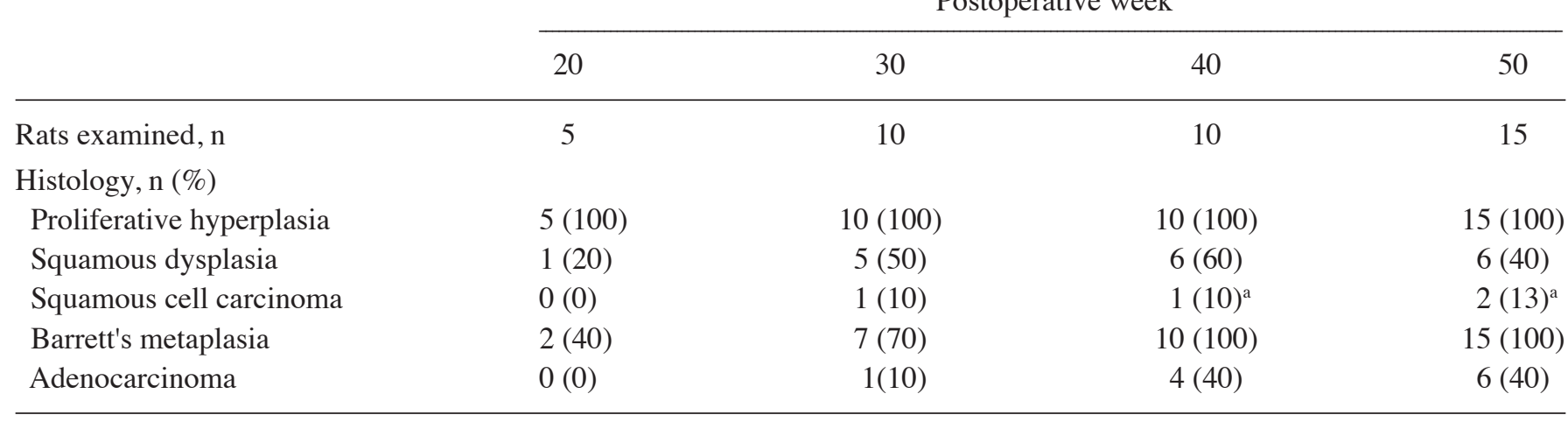

${ }^{a}$ One rat exhibited two types of carcinoma.

i) Proliferative squamous hyperplasia (PHP), a condition characterized by a thickened epithelium to twice that of a normal epithelium, with acanthosis, elongation of the papillae and parakeratosis, as well as thickening of the basal layer of the squamous epithelium and preservation of a stratified appearance; ii) squamous dysplasia, characterized by an epithelium composed of dysplastic squamous cells with large and polymorphic nuclei with deeply stained chromatin and an increased number of mitotic figures, which may involve the lamina propria of the epithelium, however, do not invade the submucosal layer; iii) Barrett's metaplasia (BM), presents replacement of the esophageal squamous epithelium with columnar-lined epithelium comprised of gastric and/or intestinal cells; and iv) carcinoma, defined as cellular and structural atypism with epithelial invasion into the submucosal layer. ADC consists of dysplastic glandular cell growth, with atypia and invasiveness, and exhibits two types of histology: Tubular or papillary ADC; and mucinous ADC. SCC is a type of squamous cell dysplasia with marked cellular and structural atypism, which may be divided into well- and poorly-differentiated types according to the presence or absence of cancer pearls, respectively.

\section{Results}

Histological findings. In total, 40/50 rats survived following the surgery and were used in the present study. Immunohistochemistry was performed (Fig. 1) and demonstrated normal esophageal epithelium in the upper esophagus at 20 weeks post-surgery (Fig. 1A), as well as squamous PHP (Fig. 1D), dysplasia and BM. At 30-50 weeks following surgery, 13/35 (37\%) rats had developed esophageal cancer. In addition, SCC (Fig. 1G) was observed in 4/35 (11\%) of the rats. By contrast, dysplastic changes, including BM (Fig. 1J) and ADC (Fig. 1M), were observed within 30 weeks and increased sequentially to 100 and $40 \%$, respectively at 40-50 weeks (Table I).

MTA1 expression. A high positive expression of MTA1 was identified in the basal layer of PHP (Fig. 1E), but not in the normal epithelium at 20 weeks (Fig. 1B). At 30 weeks, BM showed a high positive expression of MTA1 (Fig. 1K). ADC-associated BM showed a high positive expression of
MTA1 at 30-50 weeks post-surgery (Fig. 1N). By contrast, SCC demonstrated marginally reduced expression of MTA1 at 30-50 weeks post-surgery (Fig. $1 \mathrm{H})$. The MTA1 immunohistochemistry staining showed nuclear expression of MTA1 in all of the stages of squamous carcinogenesis, including PHP, squamous dysplasia and SCC, and adenocarcinogenesis, including BM and ADC, however, this was not observed in the normal squamous epithelium (Fig. 2).

HDACl expression. Similarly, the positive expression of HDAC1 was found in PHP (Fig. 1F), BM (Fig. 1L), ADC (Fig. 1O) and SCC (Fig. 1I), but not identified in the normal squamous epithelium (Fig. 1C). Furthermore, the HDAC1 immunohistochemical staining showed nuclear expression of HDAC1 in all of the stages of squamous carcinogenesis, including PHP, squamous dysplasia and SCC, and adenocarcinogenesis, including BM and ADC, however, this was not observed in the normal squamous epithelium (Fig. 2).

\section{Discussion}

The incidence of GERD-induced esophageal carcinoma is rising in the USA and the Western world $(4,5)$. We have pioneered the use of a rat reflux model of esophageal carcinoma, which is based on surgically inducing duodenogastroesophageal reflux akin to GERD in humans without the use of a carcinogen (8-10). The model has been successfully used to investigate reflux-induced esophageal carcinogenesis. While the correlation between reflux and esophageal carcinoma has been investigated in a number of studies, the molecular mechanisms underlying esophageal carcinogenesis remains poorly understood (8-10).

Numerous molecular alterations leading to the development of esophageal carcinoma have been reported (12). Chronically inflamed tissue results in the activation of multiple signaling pathways that lead to inflammation and tumorigenesis. A number of these factors, such as NF- $\mathrm{B}$ and Egr-1, have been shown to have additive or synergistic effects on the activation of a number of inflammation-associated genes, particularly those that are associated with the neoplastic transformation (25).

The alterations of the chromatin structure by HATs and $H D A C s$ are implicated in the regulation of gene transcription, as well as in the process of carcinogenesis. Tumors demonstrate 

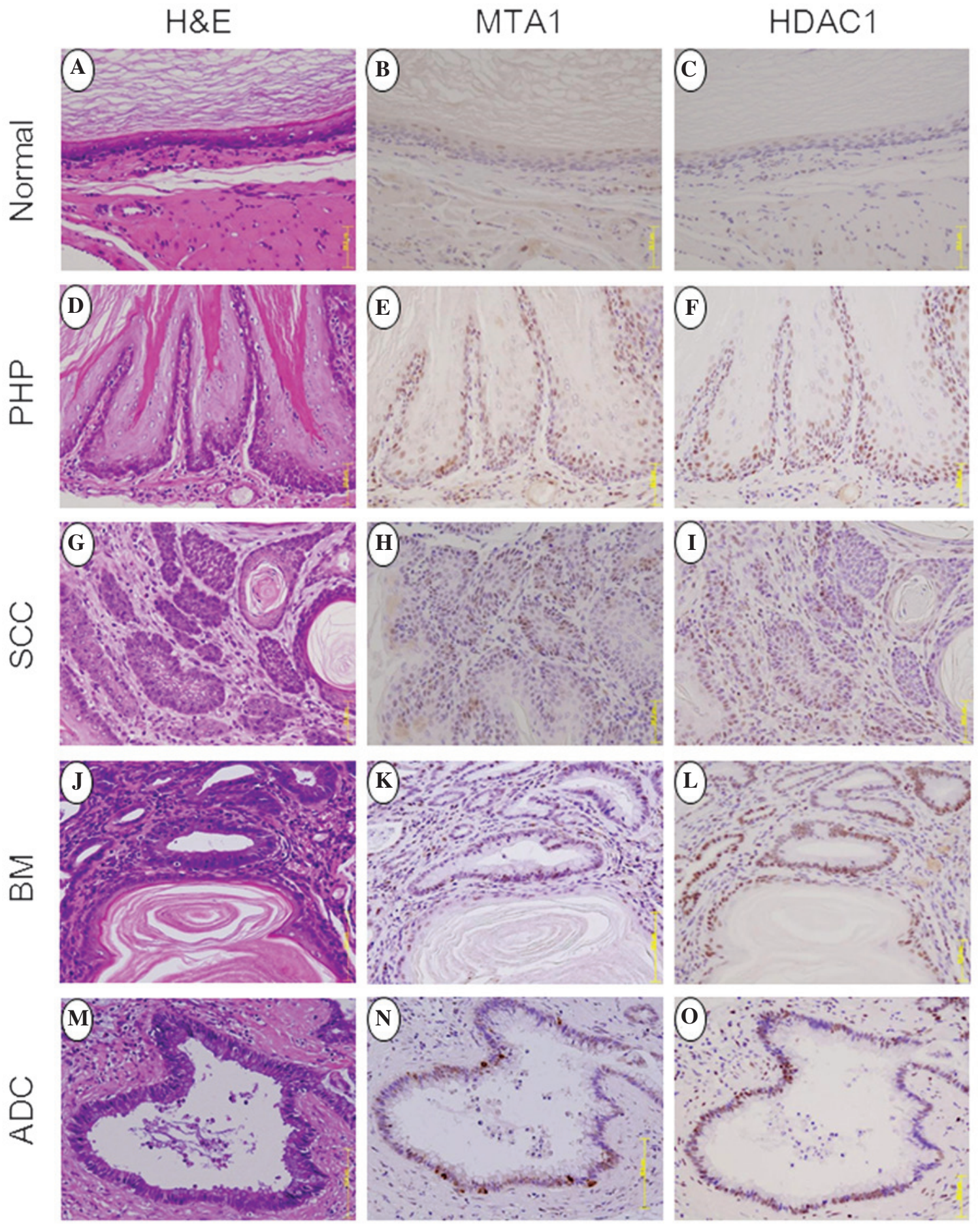

Figure 1. (A) Normal squamous epithelium did not stain for (B) MTA1 or (C) HDAC1. (D) PHP showing papillary projections and hyperkeratosis with (E) MTA1 and (F) HDAC1 expression in the two to three basal layers of papillary hyperplasia. (G) Pure SCC showing dysplastic squamous cells with marked structural atypism and cancer pearls were stained positive for (H) MTA1 and (I) HDAC1. (J) BM surrounded by a squamous lesion expressing (K) MTA1 and (L) HDAC1 in the nucleus. (M) Pure ADC showing dysplastic glandular cell growth with atypia and invasiveness stained positive for (N) MTA1 and (O) HDAC1 expression in the nucleus. H\&E, hematoxylin and eosin; MTA, metastasis-associated gene; HDAC, histone deacetylase; PHP, proliferative squamous hyperplasia; SCC, squamous cell carcinoma; BM, Barrett's metaplasia; ADC, adenocarcinoma.

the hyperacetylation of histone $\mathrm{H} 4$ and the increased expression of $H D A C l$, thus implying that a certain interaction may exist between the hyperacetylation of histone $\mathrm{H} 4$ and $H D A C l$ expression (21). $\triangle \mathrm{Np} 63 \alpha$, an N-terminally truncated form, which functions as a key ESCC cell survival factor, associates with $H D A C 1$ and $H D A C 2$ to form an active transcriptional repressor complex that may be targeted to provide a therapeutic advantage.
The repression of the proapoptotic Bcl-2 family member genes, including $p 53$ upregulated modulator of apoptosis, by $p 63 / H D A C$ is required for the survival of ESCC cells (26). Therefore, the immunohistochemical determination of HDACs may aid with predicting the response to specific HDAC inhibitors (27).

$M T A$ is a newly discovered family of cancer progression-associated genes (13). MTA1, the first gene identified in 


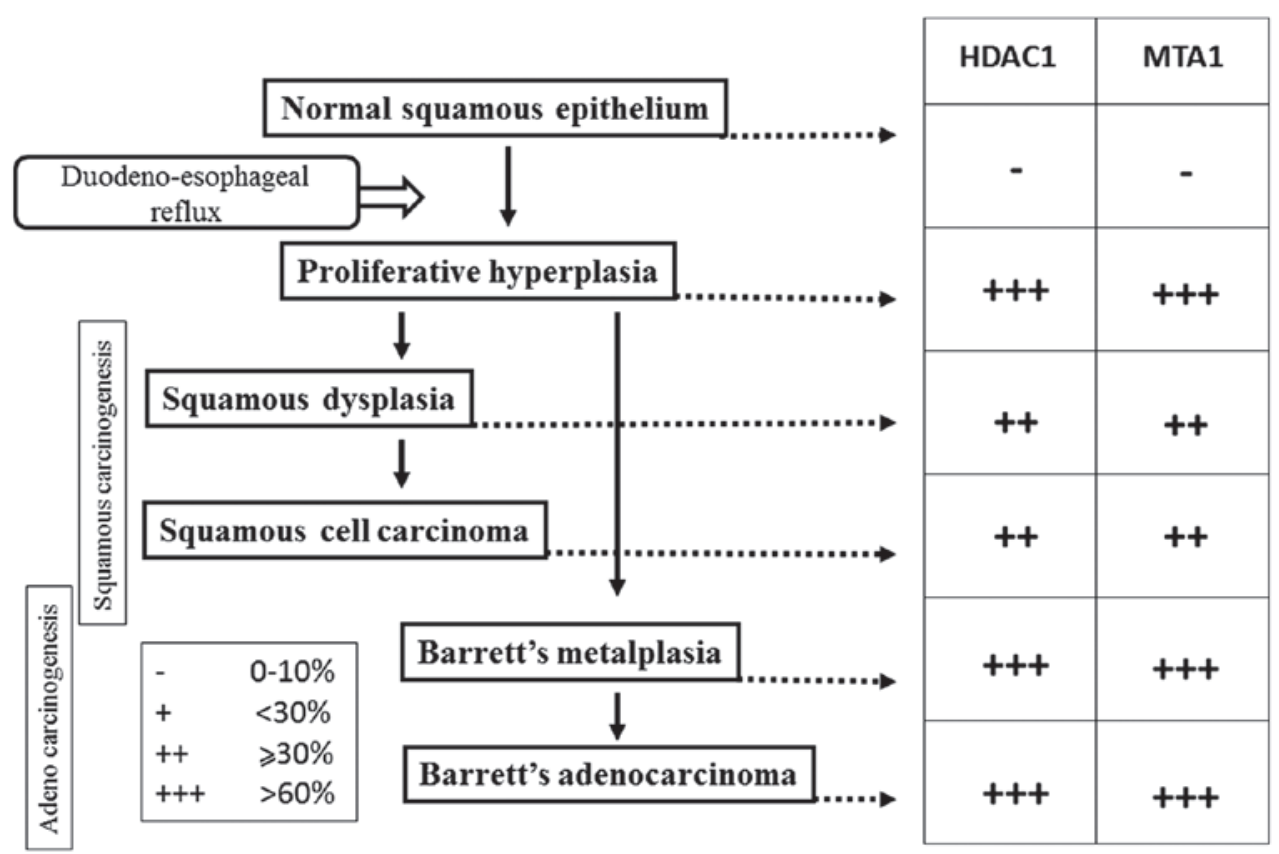

Figure 2. Immunohistochemical pattern of MTA1 and HDAC1 expression through the progression from normal epithelium to different types of carcinoma. HDAC1, histone deacetylase 1; MTA1, metastasis-associated gene 1 .

this family, has been repeatedly reported to be overexpressed along with its protein product, MTA1, in a wide range of human cancers. Therefore, MTA1 may be one of the significant molecules in cancer progression. Esophageal cancers have been investigated for MTA1/MTA1 overexpression and those esophageal cancer cells that were overexpressing MTA1/MTA1 have shown significantly higher frequencies of adventitial invasion and lymph node metastasis, as well as higher rates of lymphatic involvement (28). Thus, the MTA1 protein may be a useful predictor of the malignant potential of ESCC (14).

ATP-dependent chromatin-remodeling complexes open chromatin structures and facilitate transcriptional activation. A novel human complex, NURD, contains ATP-dependent nucleosome remodeling activity and HDAC activity that associate with transcriptional repression. The NuRD complexes share four core proteins (HDAC1, HDAC2, RbAp46 and RbAp48) with the Sin 3 complex. These complexes contain the dermatomyositis-specific autoantigens, Mi- $\alpha / \beta, \mathrm{MTA} 1 / 2$ and p66, which are functionally and physically linked (29-33).

The MTA1 protein, a component of the NuRD complex, possesses strong transcription-repressing activity (30). MTA2, another component of the NuRD complex, is highly expressed in rapidly dividing cells (32). Toh et al (34) reported the physical interaction between the MTA1 protein and HDAC1. The MTA protein family members basic functions of chromatin remodeling and histone deacetylating activities are exerted via NuRD complexes. While there are additional non-histone deacetylating proteins in NuRD complexes, the MTA proteins are likely to be the principal components. In addition, the MTA-NuRD complexes show transcription-repression activities $(35,36)$. Although all MTA protein family members are found in NuRD complexes, each MTA protein may form a distinct NuRD complex that targets different sets of promoters (33). Thus, the MTA-HDAC complex is further involved in the normal transcriptional balance of the cell (37).

HDAC, via NuRD complexes containing MTA proteins, deacetylates, chromatin histones and non-histone proteins. The p53 tumor suppressor protein was the first non-histone protein reported to be deacetylated by MTA protein-containing NuRD complexes $(23,37)$. A HDAC1 complex, which contains the MTA2 protein mediates the deacetylation of $\mathrm{p} 53$. An MTA2-associated NuRD complex is also involved, and this HDAC1/MTA2 complex interacts with p53 in vitro and in vivo, which significantly reduces the steady-state levels of acetylated p53. The deacetylation of p53 causes an increase in its own degradation through MDM2 and a reduction in p53-dependent transcriptional activation. Eventually, this results in the repression of the normal p53 function that mediates cell growth arrest and apoptosis $(23,38)$. The same phenomenon is observed between the p53 and MTA1 complex $(23,24)$. Previous studies have determined that the HDAC1/MTA1 complexes deacetylate the $\mathrm{p} 53$ protein and attenuate the transactivation function of p53 in human carcinoma, thereby inhibiting p53-induced apoptosis $(18,24)$. The MTA proteins have been shown to be ubiquitinated transcriptional corepressors, which function in HDAC and are part of the NuRD complex, a nucleosome remodeling and HDAC complex whose stability appears to be regulated by the binding of ubiquitinated MTA1 to E3 ubiquitin ligase constitutive photomorphogenesis protein-1 $(37,39)$. The MTA1 protein inhibits p53-induced apoptosis by deacetylation of p53, which may be associated with the increased metastatic potential of cancer cells with high MTA1 expression $(23,24)$.

Miyatani et al (40) examined a possible association between HDAC1 and MTA1 expression, and disease progression in the esophageal metaplasia-dysplasia-carcinoma sequence and particularly in low-grade dysplasia. The percentage of HDAC1- and MTA1-positive expression in low-grade dysplasia of BM was as high as that of cancer. Therefore, BM 
with increased HDAC1 and MTA1 expression is considered to be a precancerous lesion. The results of the current study showed that MTA1 and HDAC1 expression is already present in PHP and BM. The positive expression rate of MTA1 and HDAC1 was similar to that among PHP, BM and EADC. Thus, increased MTA1 and HDAC1 expression indicates that PHP and BM may already have malignant potential.

Bonde et al (12) established that cell lines derived from a reflux-associated rat model of esophageal cancer exhibited glandular features. While the histology of the xenografts expressed a squamous phenotype when they were transplanted into nude mice. Cytogenetic analysis also showed significant similarities between rat and human esophageal cancers, including ESCC and EADC. Furthermore, cytogenetic analysis of the rat model revealed a highly aneuploid cell population with the derangement of key chromosomes that encode a variety of oncogenes. The neoplastic nature, gene expression profile and pathway activation of these rodent reflux-induced tumors was found to be comparable with human esophageal cancer. In rats with BM and EADC, the deletion and translocation of chromosome 7 and 11, and overexpression of important peptide mediators are considered to be significant in carcinogenesis, including hypoxia-inducible factor (HIF) $1 \alpha$, cyclin dependent kinase 4, vascular endothelial growth factor, polo-like kinase and the epidermal growth factor receptor.

HIF1 $\alpha$ is a key regulator of angiogenic factors (41) and another important non-histone protein that is deacetylated by the HDAC1/MTA1 complexes. The MTA1 protein binds to and deacetylates HIF1 $\alpha$, which increases HDAC1 expression and subsequently stabilizes HIF1 $\alpha$ via a positive feedback mechanism (23). The expression of HDAC1 and MTA1 is similar in squamous carcinogenesis and adenocarcinogenesis. Therefore, esophageal squamous and adeno differentiation may possess a common genetic pathway.

Histologically, the spectrum of esophageal cancer is divided into ESCC and EADC. However, the mechanism by which each histological type of carcinoma arises from the esophageal mucosa remains unknown. In our previous study, the rodents received one of the following procedures: Duodeno-forestomach reflux with reduced exposure to duodenal contents; duodenoesophageal reflux with increased exposure to duodenal contents; or three control operations. The fraction of ESCC in the reduced exposure group was significantly higher than that of the high exposure group, while the fraction of EADC in the reduced exposure group was significantly lower than that of the high exposure group. In brief, high exposure to duodenal contents promotes the development of EADC, and low exposure induces ESCC. The severity of the duodenoesophageal reflux in rodents is associated with the development of different histological types of esophageal carcinoma (11). In the present study, ESCC was shown to arise from dysplastic changes to squamous cells that were naturally located proximal to the columnar-lined epithelium. By contrast, EADC arose in the areas of columnar-lined epithelium adjacent to the surgical anastomosis.

MTA1 expression levels are closely associated with the degree of malignant potential. For example, metastatic malignant tissue demonstrates increased expression when compared with tissue from high-grade prostatic intraepithelial neoplasia (42). In the present study, squamous PHP was found to express MTA1 and HDAC1 and therefore, PHP may have malignant potential, which chemopreventive agents could be directed against.

The present study was not designed to completely evaluate the esophageal cancer signaling pathway. However, the results are consistent with those of previous studies, which demonstrate that the MTA1/HDACl complexes are involved in other malignancies. This study also demonstrates the involvement of these complexes in esophageal cancer.

Thus, it has been proposed that the MTA and HDAC proteins, particularly MTA1 and HDAC1, present as master coregulatory molecules involved in esophageal carcinogenesis.

The HDAC inhibitors have been shown to be potent inducers of growth arrest, differentiation and/or apoptotic cell death of transformed cells and, as a result, are currently receiving considerable attention as antitumor agents. In addition, the HDAC inhibitors have been reported to disrupt the cell cycle in the G2 phase, allowing cells to prematurely enter the $\mathrm{M}$ phase, as well as to interfere directly with the mitotic spindle checkpoint (43).

Kai et al (20) reported a novel pathway of chromatin remodeling by resveratrol (Res) via the functional restriction of the MTA1/NuRD complex; Res decreased MTA1 expression, thus deregulating the MTA1/HDAC1 complexes leading to increased p53 acetylation (Ac-p53), and enhanced binding to the p21 and Bax promoters in the PCa cells. Furthermore, Res treatment on MTA1-null background resulted in a marked increase in the apoptotic function of Res, which indicates MTA1 as an antiapoptotic protein. Finally, the combined treatment of Res and the HDAC inhibitor, SAHA, cooperatively increased Ac-p53 levels and apoptosis, indicating novel therapeutic strategies in combination with the use of HDAC inhibitors that have already been clinically approved (20). Thus it is necessary to administer the HDAC inhibitor and Res for the treatment of GERD patients at an early stage.

In conclusion, the expression of HDAC 1 and MTA1 may be required to promote the development of neoplastic transformation in cancer cells with squamous and adeno differentiation. Furthermore, the HDAC inhibitor and administration of Res may prevent esophageal carcinogenesis.

\section{References}

1. Hongo M, Nagasaki Y and Shoji T: Epidemiology of esophageal cancer: Orient to occident. Effects of chronology, geography and ethnicity. J Gastroenterol Hepatol 24: 729-735, 2009.

2. Wheeler JB and Reed CE: Epidemiology of esophageal cancer. Surg Clin North Am 92: 1077-1087, 2012.

3. Bollschweiler E, Wolfgarten E, Gutschow C and Hölscher AH: Demographic variations in the rising incidence of esophageal adenocarcinoma in white males. Cancer 92: 549-555, 2001.

4. Reid BJ, Li X, Galipeau PC and Vaughan TL: Barrett's oesophagus and oesophageal adenocarcinoma: time for a new synthesis. Nat Rev Cancer 10: 87-101, 2010.

5. Engel LS, Chow WH, Vaughan TL, et al: Population attributable risks of esophageal and gastric cancers. J Natl Cancer Inst 95: 1404-1413, 2003.

6. Lagergren J, Bergström R, Lindgren A and Nyrén O: Symptomatic gastroesophageal reflux as a risk factor for esophageal adenocarcinoma. N Engl J Med 340: 825-831, 1999.

7. Jankowski JA, Harrison RF, Perry I, Balkwill F and Tselepis C: Barrett's metaplasia. Lancet 356: 2079-2085, 2000.

8. Miwa K, Segawa M, Takano Y, et al: Induction of oesophageal and forestomach carcinomas in rats by reflux of duodenal contents. Br J Cancer 70: 185-189, 1994. 
9. Miwa K, Sahara H, Segawa M, et al: Reflux of duodenal or gastro-duodenal contents induces esophageal carcinoma in rats. Int J Cancer 67: 269-274, 1996.

10. Miyashita T, Ohta T, Fujimura T, et al: Duodenal juice stimulates oesophageal stem cells to induce Barrett's oesophagus and oesophageal adenocarcinoma in rats. Oncol Rep 15: 1469-1475, 2006.

11. Miyashita T, Miwa K, Fujimura T, et al: The severity of duodeno-esophageal reflux influences the development of different histological types of esophageal cancer in a rat model. Int J Cancer 132: 1496-1504, 2013.

12. Bonde P, Sui G, Dhara S, et al: Cytogenetic characterization and gene expression profiling in the rat reflux-induced esophageal tumor model. J Thorac Cardiovasc Surg 133 763-769, 2007.

13. Nawa A, Nishimori K, Lin P, et al: Tumor metastasis-associated human MTA1 gene: its deduced protein sequence, localization, and association with breast cancer cell proliferation using antisense phosphorothioate oligonucleotides. J Cell Biochem 79: 202-212, 2000.

14. Toh Y, Ohga T, Endo K, et al: Expression of the metastasis-associated MTA1 protein and its relationship to deacetylation of the histone $\mathrm{H} 4$ in esophageal squamous cell carcinomas. Int J Cancer 110: 362-367, 2004

15. Toh Y, Pencil SD and Nicolson GL: Analysis of the complete sequence of the novel metastasis-associated candidate gene, mta1, differentially expressed in mammary adenocarcinoma and breast cancer cell lines. Gene 159: 97-104, 1995.

16. Iguchi H, Imura G, Toh Y and Ogata Y: Expression of MTA1, a metastasis-associated gene with histone deacetylase activity in pancreatic cancer. Int J Oncol 16: 1211-1214, 2000.

17. Higashijima J, Kurita N, Miyatani T, et al: Expression of histone deacetylase 1 and metastasis-associated protein 1 as prognostic factors in colon cancer. Oncol Rep 26: 343-348, 2011.

18. Nicolson GL, Nawa A, Toh Y, Taniguchi S, Nishimori K and Moustafa A: Tumor metastasis-associated human MTA1 gene and its MTA1 protein product: role in epithelial cancer cell invasion, proliferation and nuclear regulation. Clin Exp Metastasis 20: 19-24, 2003.

19. Moon WS, Chang K and Tarnawski AS: Overexpression of metastatic tumor antigen 1 in hepatocellular carcinoma: Relationship to vascular invasion and estrogen receptor-alpha. Hum Pathol 35: 424-429, 2004

20. Kai L, Samuel SK and Levenson AS: Resveratrol enhances p53 acetylation and apoptosis in prostate cancer by inhibiting MTA1/NuRD complex. Int J Cancer 126: 1538-1548, 2010.

21. Toh Y, Yamamoto M, Endo K, et al: Histone H4 acetylation and histone deacetylase 1 expression in esophageal squamous cell carcinoma. Oncol Rep 10: 333-338, 2003.

22. Sun WJ, Zhou X, Zheng JH, et al: Histone acetyltransferases and deacetylases: molecular and clinical implications to gastrointestinal carcinogenesis. Acta Biochim Biophys Sin (Shanghai) 44: 80-91, 2012.

23. Toh Y and Nicolson G: MTA1 of the MTA (metastasis-associated) gene family and its encoded proteins: molecular and regulatory functions and role in human cancer progression. Atlas Genet Cytogenet Oncol Haematol 15: 303-315, 2011.

24. Moon HE, Cheon H and Lee MS: Metastasis-associated protein 1 inhibits p53-induced apoptosis. Oncol Rep 18: 1311-1314, 2007.

25. Abdel-Latif MM, Duggan S, Reynolds JV and Kelleher D Inflammation and esophageal carcinogenesis. Curr Opin Pharmacol 9: 396-404, 2009
26. Ramsey MR, He L, Forster N, Ory B and Ellisen LW: Physical association of HDAC1 and HDAC2 with p63 mediates transcriptional repression and tumor maintenance in squamous cell carcinoma. Cancer Res 71: 4373-4379, 2011

27. Langer R, Mutze K, Becker K, et al: Expression of class I histone deacetylases (HDAC1 and HDAC2) in oesophageal adenocarcinomas: an immunohistochemical study. J Clin Pathol 63: 994-998, 2010.

28. Toh Y, Kuwano H, Mori M, Nicolson GL and Sugimachi K: Overexpression of metastasis-associated MTA1 mRNA in invasive oesophageal carcinomas. Br J Cancer 79: 1723-1726, 1999.

29. Tong JK, Hassig CA, Schnitzler GR, Kingston RE and Schreiber SL: Chromatin deacetylation by an ATP-dependent nucleosome remodelling complex. Nature 395: 917-921, 1998.

30. Xue Y, Wong J, Moreno GT, Young MK, Côté J and Wang W: NURD, a novel complex with both ATP-dependent chromatin-remodeling and histone deacetylase activities. Mol Cell 2: 851-861, 1998

31. Wade PA, Gegonne A, Jones PL, Ballestar E, Aubry F and Wolffe AP: Mi-2 complex couples DNA methylation to chromatin remodelling and histone deacetylation. Nat Genet 23: 62-66, 1999.

32. Zhang Y, Ng HH, Erdjument-Bromage H, Tempst P, Bird A and Reinberg D: Analysis of the NuRD subunits reveals a histone deacetylase core complex and a connection with DNA methylation. Genes Dev 13: 1924-1935, 1999.

33. Bowen NJ, Fujita N, Kajita M and Wade PA: Mi-2/NuRD: multiple complexes for many purposes. Biochim Biophys Acta 1677: 52-57, 2004

34. Toh Y, Kuninaka S, Endo K, et al: Molecular analysis of a candidate metastasis-associated gene, MTA1: possible interaction with histone deacetylase 1. J Exp Clin Cancer Res 19: $105-111,2000$

35. Kumar R, Wang RA and Bagheri-Yarmand R: Emerging roles of MTA family members in human cancers. Semin Oncol 30 (Suppl 16): S30-S37, 2003

36. Manavathi B, Peng S, Rayala SK, et al: Repression of Six3 by a corepressor regulates rhodopsin expression. Proc Natl Acad Sci USA 104: 13128-13133, 2007.

37. Toh Y and Nicolson GL: The role of the MTA family and their encoded proteins in human cancers: molecular functions and clinical implications. Clin Exp Metastasis 26: 215-227, 2009.

38. Luo J, Su F, Chen D, Shiloh A and Gu W: Deacetylation of p53 modulates its effect on cell growth and apoptosis. Nature 408: $377-381,2000$

39. Li DQ, Ohshiro K, Reddy SD, et al: E3 ubiquitin ligase COP1 regulates the stability and functions of MTA1. Proc Natl Acad Sci USA 106: 17493-17498, 2009

40. Miyatani T, Kurita N, Mikami C, et al: Malignant potential of Barrett's esophagus: special reference to HDAC-1 and MTA-1 expression. Hepatogastroenterology 58: 472-476, 2011.

41. Yoo YG, Na TY, Seo HW, et al: Hepatitis B virus X protein induces the expression of MTA1 and HDAC1, which enhances hypoxia signaling in hepatocellular carcinoma cells. Oncogene 27: 3405-3413, 2008.

42. Hofer MD, Kuefer R, Varambally S, et al: The role of metastasis-associated protein 1 in prostate cancer progression. Cancer Res 64: 825-829, 2004

43. Taddei A, Roche D, Bickmore WA and Almouzni G: The effects of histone deacetylase inhibitors on heterochromatin: implications for anticancer therapy? EMBO Rep 6: 520-524, 2005. 